\title{
What We Can Learn in a Pandemic
}

\section{Hoffman DP*}

Department of Ethics and Health Policy, Maria College, USA

*Corresponding author: David P Hoffman, DPS CCE, Associate Dean for Academic Initiatives and Government Affairs, Department of Ethics and Health Policy, Maria College 700 New Scotland Ave, Albany, New York 12208, USA, Tel: 5183667544; Email: dhoffman@mariacollege. edu

\section{Editorial}

This Novel Coronavirus/SARS CoV2 pandemic has been eye opening in many ways. We've seen some countries and jurisdictions use public health, science and the application of principles of bioethics and exert control in ways that limit the human carnage that is all too evident in others. For example, the data coming from South Korea indicate that in a population of 51.61million 26,807people have been infected and 472 died. At the same time in the US with a population of 326.7million (about 6.3times that of So Korea), 9,296,159 people have been infected and 231,591 (about 491 times that of South Korea) have died. Note that if the rate remained the same at the population U.S deaths would be in the range of 2,974 (source: https://coronavirus.jhu.edu/map.html ; accessed November 3, 2020 at 10:30 am Eastern time).

A number of years ago I was introduced to the concept of "excess death", that is those deaths that occur beyond what are predictable in a population. The context at the time was studying the impact of social determinants of health and the presence of chronic disease which often are impacted by behavior and level of stress; these to be sure are behind a large discrepancy in the difference between predictable deaths and actual deaths. In the context of the SARS CoV2 pandemic we are seeing a different factor impact the rate of excess death. This factor reflects the public health approach as impacted by leadership and media which in turn impact the beliefs and actions of populations and the organizations they interact with in their daily lives. In this regard the actions in South Korea and the US are a stark comparison when filtered through a lens of Beauchamp and Childress principles of bioethics.

According to a piece in Medical News Today from August there are identifiable factors that lead to the South Korean success in addressing the pandemic [1]. These include having a well-prepared plan that is updated periodically, relying on a comprehensive contact tracing system, and flexibility in health systems. This was supported and led by clear, concise and open communication from government officials and private partners that included opportunity for regular questions for experts and dialogue with press and others supporting confidence in the approach and underpinning of science.

The Korean Ministry of Health and Welfare (KMHW) maintains an infectious disease plan that is updated through a process every five years. This process encourages participation and confidence among public health and healthcare professions and the media. In doing so the support for what are otherwise unpopular actions are understood and supported because diverse parties understand the value. The inclusion of private sector collaboration enhanced South Korea's ability to respond with robust testing capacity through development of tests in collaboration with private industry and then turning the testing technology over to private industry with capacity to rapidly manufacture kits that in turn were distributed to national and local public health authorities.

By comparison the US response to Novel Coronavirus/ SARS CoV2 pandemic has been catalogued by various media so often and so much that I'm challenged to find clear sources to share. This is part of the issue; the conflicting reports on the US response show a sharp contrast to that of South Korea. The lack of a clear agreement on what happened, why, when and how is all part of the explanation for the complicated situation we see when attempting to learn from this ongoing crisis. In some sense numbers speak for themselves, but in bioethics we also recognize that each of those numbers represent a person [2]. 
It is for this reason I look back and forward to the principles of bioethics offered to us first 40years ago by Beauchamp and Childress in Principles of Bioethics as a filter to view our reality [3]. My hope is to use these principles to illuminate certain key elements for public and public health officials of the future to aim for more impactful strategy and inclusive understanding of public health methods and priorities that track back through history. We often use the example of Dr John Snow in London during the cholera outbreak of 1854 . It has often occurred to me that if John Snow's action removing the pump handle from the public water supply likely made him an unpopular individual in the community in spite of the lives he saved. Today we have many additional tools and using the four principles of bioethics can help us see the utility of actions even when they are sometimes unpopular.

The first principle, autonomy, is sometimes simplified in a way that can change the meaning. The principal calls for respect for the autonomy of the individual. But it also presumes that the individual has capacity with understanding and is making voluntary decisions. Unpacking that helps us to understand that we each have autonomy about decisions that impact our body, but that we need to have capacity to understand all the implications for ourselves and others and all relevant information to round out that understanding. The exercise of autonomy is dramatically compromised in a culture where information can be weaponized and terms like "fake news" and "deep state" are used to describe previously universally trusted sources of information. This leads to less than full and accurate information for people to use to make individual healthcare decisions and public officials, leaders of business and other organizations to establish their own relevant policies. So here in the US right now our political differences negatively impact our health.

The second principle, beneficence, calls for all in healthcare to "do good" and work to prevent harm in all our actions. If we apply this to public health the goal remains the same. Our public health actions whether for prevention or to ameliorate a crisis should be aimed at doing good. While this may sound pretty simple, in a world where mistrust underlies much of the public discourse and animus is attributed to those who oppose our views, achieving trust to accomplish good through policies that in a pandemic by definition can have negative impacts on business and social life is a very complicated matter. Beneficence often looked to as the principle of bioethics easiest to understand and initiate has become a challenge in the world of public health in this time of pandemic. In public health and public policy related to health we should always have confidence that the motivation behind a recommendation or action includes beneficence. This confidence can help everyone work toward common goals of protecting and improving health for all.
The absence of this confidence can cause us to focus inward, selfishly ignoring risk to others while seeking benefit for ourselves.

The third and arguably most famous principle, nonmaleficence in the vernacular, means "first does no harm". In individual health this concept is sometimes clearer than in public health. If the action of my provider doesn't make me worse and helps with a problem or issue it may well be ethical. It is important to remember that this principle relates to both acts of commission and acts of omission. In the context of a pandemic when we address the public with less than the full story to save or support an issue we see as important we may well cause harm. I'm sure each of us can recall examples in the past few months that illustrate this concept. My point is those examples where either the full story isn't told, or the partial story leads to incorrect conclusions are clear violations of this principle. Additionally, when we prioritize other values over the health of a population (i.e. profit) we violate this principle as we cause avoidable risk, or known danger by dismissing or ignoring the best science of the time.

The last principle is Justice, often described as fairness for all. This implies the fair distribution of goods and services in a society. This "distributive justice" recognizes that there are sometimes shortages of important resources (especially in health) and that calls for a fair method of distribution. Some of the criteria commonly used include:

- An equal share for everyone

- Meeting the needs of each person

- Rewarding people based on effort

- Recognizing merit in everyone

When we consider these statements, we recognize that each brings with it certain value judgments on what fairness means. Fairness can mean different things to each of us, but when we apply fairness or justice standard to health resources, we must understand that we also need to collectively define that standard, or we are using the same words to have different meanings. I'm also sure when we each look at the availability of healthcare services during a pandemic it is easy to see that these concepts quickly come to challenge by the reality of limitations in supply of both material and human resources. How often have we heard and read about hospitals with full Intensive Care Units or building auxiliary hospitals in arenas or other facilities. Does anyone assume the quality of care is equal in a sports stadium converted to a medical facility and an Intensive Care Unit in a hospital constructed to strict standards just for that purpose? Should we assume when we hear that some high level official or wealthy/famous person tested positive is getting the latest in treatment protocols that the same is available to all? The goal of fairness and justice in health is one we can spend volumes discussing. We clearly have work to do in this arena, 
and the presence of a pandemic only complicates the issues and multiples the impact. It is for these reasons we need to refocus on justice and fairness in health and healthcare and use justice and fairness as filters when we look at public health strategies to address this crisis. This also requires we engage in open honest and comprehensive dialogue with fellow professionals and the public.

The South Korean approach of developing a plan with broad input before predictable crisis appears to have put them in an advantageous position to address the pandemic, at least at this point. The planning process, perhaps unintentionally, causes participants to consider relevant issues including those related to autonomy, beneficence, non-maleficence, and justice. In doing participants and those educated about the process can support implementation with confidence. There are sharp contrasts to this experience in other countries and regions. Other countries can learn from this example potentially to address then rest of this crisis or prepare for the predictable crisis of the future.

Meanwhile we can still take our own individual actions based on these same lessons and principles. There are enough examples in communities across the world of people whose surrogate decision maker is banned from the facility where they are receiving treatment due to the pandemic and the safety of everyone, including the surrogate. That just makes the recommendations below for each of us more important when considering the reality we live in. Here are as my recommendations that follow the principles above.
- $\quad$ First make a plan. Ask yourself what you want to happen to you if challenging circumstances challenge your health or life. There are many stories already about what has happened to people since this pandemic began don't pretend those things can't happen they already have. So base your plan on what is important to you.

- Second, write it down. In many places there are forms and formats to express your wishes available on-line and otherwise. Use one and be clear in your wishes.

- Third, talk about it. Discussing our health and morality doesn't come naturally to many of us but we must realize that it is often someone else who ends up making important decisions for us.

It is more likely our wishes will be understood, respected and carried out if we have that conversation with people likely to be our decision makers if we're not able to express our wishes ourselves. Lastly, use the principles I describe above as filters to illustrate your wishes. This can make them easier to understand and comforting for others to carry out.

\section{References}

1. (2020) Medical News Today. Timothy Huzar

2. You J (2020) Lessons from South Korea's Covid-19 Policy Response. Sage Journals 50(6-7): 801-808.

3. Beauchamp T, Childress J (2019) Principles of Biomedical Ethics. $8^{\text {th }}$ (Edn.), Oxford University Press NY.

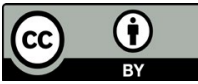

